\title{
Using patient decision aids to promote evidence-based decision making
}

Evidence-based medicine integrates clinical experience with patients' values and the best available evidence. ${ }^{1}$ In the past, clinicians took responsibility not only for being well informed about the benefits and harms of medical options but also for judging their value in the best interests of the patients. More recently, a shared decision making approach has been advocated in which patients are recognised as the best experts for judging values. Evidence-based decision aids are being developed and evaluated to supplement clinicians' counselling regarding values and sensitive options so that patients can understand the probable consequences of options, consider the value they place on the consequences, and participate actively with their clinician in selecting the best option for them. This editorial provides a brief overview of patient decision aids by defining them, identifying situations when they may be needed, describing their efficacy, and discussing practical issues in using them in clinical practice.

\section{What is a patient decision aid?}

Decision aids help patients to participate with their practitioners in making deliberative, personalised choices among healthcare options. The key elements of decision aids have been described by the Cochrane Collaboration ${ }^{2}$ as

\section{INFORMATION TAILORED TO THE PATIENT'S HEALTH}

STATUS

Information is provided on the condition, disease, or developmental transition stimulating the decision; the healthcare options available; the outcomes of options, including how they affect patient functioning; and the probabilities associated with outcomes.

\section{VALUES CLASSIFICATION}

Values clarification exercises are used to explicitly consider and communicate the personal importance of each benefit or harm by using such strategies as balance scales, relevance charts, or trade off techniques.

\section{EXAMPLES OF OTHER PATIENTS}

Patients often like to learn from others who have faced the same situation, and aids can give a balanced illustration of how others deliberate about options and arrive at decisions based on their personal situation.

GUIDANCE OR COACHING IN SHARED DECISION MAKING Skills and confidence in participating in decision making are developed by guiding patients in the steps involved and by discussing values and personal issues.

\section{MEDIUM OF DELIVERY}

Decision aids are delivered as self administered tools or practitioner administered tools in one to one or group sessions.
Possible media include decision boards, interactive videodiscs, personal computers, audiotapes, audio guided workbooks, and pamphlets.

This definition clearly shows that information is a necessary but not an exclusive element of decision aids. People need to learn how to personalise this information and how to communicate their personal issues and values to their practitioners. Decision aids are meant to supplement rather than to replace counselling, and follow up with a practitioner is a necessary part of providing decision support.

The Cochrane Collaboration also defines what decision aids are not. ${ }^{2}$ They do not include educational materials that inform people about health issues in general ways but that do not support decision making about a specific set of options relevant to the patient. Decision aids are not passive informed consent materials in which a clinician recommends a strategy and then provides information for providing consent. And finally, they are not interventions designed to promote compliance with a recommended option rather than a choice based on personal values.

\section{When do you need a decision aid?}

The use of decision aids is usually reserved for circumstances in which patients need to carefully deliberate about the personal value of the benefits and harms of options. Clinicians are beginning to get easy access to high quality summaries of the benefits and harms of management options in such evidence-based resources as Clinical Evidence. ${ }^{3}$ Kassirer $^{4}$ lists some indications for explicitly eliciting patients' values in clinical practice, including situations where:

- Options have major differences in outcomes or complications

- Decisions require making tradeoffs between short term and long term outcomes

- One choice can result in a small chance of a grave outcome

- There are marginal differences in outcomes between options.

Patient characteristics may also determine the need for a decision aid. For example, if patients are risk averse or if they attach unusual importance to certain possible outcomes (eg, risks for disease from blood transfusions), a decision aid might be helpful.

Another useful strategy for determining the need for a decision aid is to classify treatment policies as standards, guidelines, or options by using Eddy's definitions. ${ }^{5}$ For standards of care in which outcomes are known and patients' preferences are generally consistent in favouring an intervention, decision aids may be less useful and conventional informed consent procedures more appropriate. Examples include the use of insulin in patients with type I diabetes mellitus or the use of antibiotics in patients with an infection known to be responsive to antibiotics. 
List of Decision Aids

\begin{tabular}{lll} 
Condition specific decision aids & Delivery format & Publication \\
Breast cancer surgery & Audio cassette/booklet & Health Expectations 1998;1:23-36 \\
& & Med Decis Making 2001;21:1-6 \\
\hline Atrial fibrillation and antithrombotic treatment & Audio cassette/booklet & JAMA 1999;282:737-43 \\
& & J Gen Intern Med 2000;15:723-30 \\
\hline Cardiac ischaemic treatment & Video disc & J Gen Intern Med 2000;15:685-93 \\
& & J Gen Intern Med 1996;11:373-6 \\
\hline Menopause options & Patient Educ Counsel 1998;33:267-79 \\
& Audio cassette/booklet & Med Decis Making 1998;18:295-303 \\
\hline Benign prostatic hyperplasia treatment & Video/video disc & Med Care 1995;33:765-70 \\
\hline Prostate specific antigen testing & & J Gen Intern Med 1996;11:342-9 \\
& Video cassette/booklet & Arch Fam Med 1999;8:333-40
\end{tabular}

In contrast, when a treatment policy is classified as a guideline or an option, decision aids may be indicated because outcomes may be less certain or values for the benefits relative to the harms are more variable or unknown. For example, good evidence exists that amniocentesis performed on pregnant women who are $>35$ years of age is effective in detecting abnormalities, but not all women choose the procedure because their values about the medical options and potential outcomes differ. Benign prostatic hypertrophy is another example because it has several management options (watchful waiting, drugs, or surgery) and potential outcomes (amount of symptom relief $v$ drug side effects or surgical risks of incontinence and impotence) that each patient may value differently.

\section{Do decision aids work?}

Evaluation studies from a Cochrane systematic overview of trials $^{2}$ and 2 general reviews ${ }^{67}$ have shown that decision aids improve decision making by:

- Reducing the proportion of patients who are uncertain about what to choose

- Increasing patients' knowledge of the problem, options, and outcomes

- Creating realistic personal expectations (perceived probabilities) of outcomes

- Improving the agreement between choices and a patients' values

- Reducing some elements of decisional conflict (feeling uncertain, uninformed, unclear about values, and unsupported in decision making)

- Increasing participation in decision making without adversely affecting anxiety.

However, the impact of decision aids on satisfaction with decision making is more uncertain. More research is needed on the clinical, behavioural, and service utilisation outcomes of decisions. We also need to know which decision aids work best with which decisions and which types of patients and how acceptable decision aids are to practitioners and to diverse groups and cultures.

Questions also exist about the essential elements in decision aids. Although decision aids have been quite beneficial relative to usual care, the differences between simpler and more detailed aids have often been marginal.

\section{How do you know a particular decision aid is a} good one?

The definition of a patient decision aid is open to broad interpretation, and materials of variable quality have been produced. Consumers expect to receive free health information and may have difficulty distinguishing the wheat from the "free" chaff unless certain standards are set in their development.

A high quality decision aid should

- Be evidence-based, using evidence-based statements of benefits and risks from credible sources; refer to the quality and consistency of empirical studies; and use systematic overviews that extend shelf life and enhance updating

- Be balanced in presenting all options (including doing nothing), the benefits and risks of all options, and (when available) examples of others' decisions and opinions

- Have credible developers with expertise as evidence interpreters, communicators, practitioners, consumers, and disseminators

- Be up to date by using expiry dates indicating the expected shelf life of the information, mentioning upcoming trials that may shift policy, and demonstrating linkage to an ongoing and credible evidence analysis process (eg, the International Cochrane overview groups, the US Agency for Health Care Research and Quality evidence centres, and the Canadian Cancer Care Ontario Practice Guidelines Initiative)

- Identify conflicts of interests of developers and funding sources

- Provide evidence of evaluation describing how the aid improves decision making.

HOW DO YOU ACCESS THESE DECISION AIDS?

The Cochrane decision aids review group is assembling a list of all decision aids available and under evaluation. Most aids have been used in research environments for evaluation. The table lists the decision aids that have been developed, evaluated, and made available to others. (For a complete list of decision aids that have been developed, please see http://www.ohri.ca/ programs/clinical-epidemiology/ohdec.)

Many of the decision aids are self administered in video, computer, or audio guided workbook formats so that they can be used feasibly in settings with limited personnel and limited time for counselling. Therefore, the time that practitioners can spend on counselling focuses on personal deliberation rather than providing factual information. Some larger health services (eg, health maintenance organisations in the US) also employ telephone health coaches: patients are referred to them to access materials and to ask questions in preparation for follow up counselling with their individual practitioners. This delivery strategy may become more feasible in other countries as tele-health services expand (eg, NHS Direct in the UK and InfoSante in Quebec, Canada). 
A critical challenge during the next few years will be developing best practices for deploying those decision aids that have been validated in clinical practice so that they are used, they improve counselling, and they promote evidence-based decision making that is consistent with patients' values.

ANNETTE O'CONNOR, RN, PhD

University of Ottawa School of Nursing and Department of Epidemiology

Ottawa Health Research Institute

Ottawa, Ontario, Canada

1 Sackett DL, Rosenberg WM, Gray JA, et al. Evidence-based medicine, what it is and what it isn't. BMJ 1996;312:71-2.
2 O'Connor A, Rostom A, Fiset V, et al. Decision aids for patients facing health treatment or screening decisions: a Cochrane systematic review. BMJ 1999;319:731-4.

3 Barton S, ed. Clinical evidence. London: BMJ Publishing; 2000.

4 Kassirer JP. Incorporating patients' preferences into medical decisions. $N$ Engl J Med 1994;330:1895-6.

5 Eddy DM. A manual for assessing health practices and designing practice policies: the explicit approach. Philadelphia: American College of Physicians, 1992

6 O'Connor AM, Fiset V, DeGrasse C, et al. Decision aids for patients considering health care options: evidence of efficacy and policy implications. J Natl Cancer Inst Monogr 1999;25:67-80.

7 Molenaar S, Sprangers MA, Postma-Schuit FC, et al. Feasibility and effects of decision aids. Med Decis Making 2000;20:112-27.

\section{EVIDENCE-BASED MEDICINE LIVE AND ONLINE!}

\section{www.evidence-basedmedicine.com}

We are pleased to announce that EBM online (www.evidence-basedmedicine.com) is now available to our readers. Features of the site include:

- Full text, downloadable in PDF format

- Links to primary papers and references-full text links to >200 journals even if you don't subscribe to them

- Browse by keyword or topic

- Direct access to Medline

- Email table of contents alerts

We invite you to take a look at the web site and email us via the feedback facility with any comments.

Brian Haynes

Paul Glasziou

\section{Journals reviewed for this issue*}

Acta Obstet Gynecol Scand
Age Ageing
Am J Cardiol
Am J Med
Am J Obstet Gynecol
Am J Psychiatry
Am J Public Health
Am J Respir Crit Care Med
Am J Surg
Ann Emerg Med
Ann Intern Med
Ann Med
Ann Surg
Arch Dis Child
Arch Fam Med
Arch Gen Psychiatry
Arch Intern Med

Acta Obstet Gynecol Scand

Arch Neurol

Arch Pediatr Adolesc Med

Arch Surg

Arthritis Rheum

BMJ

Br J Gen Pract

Br J Obstet Gynaecol

BrJ Psychiatry

Br J Surg

CMAJ

Chest

Circulation

Clin Invest Med

Clin Pediatr

Cochrane Library

Crit Care Med

Diabetes Care
Fertil Steril
Gastroenterology
Gut
Heart
Hypertension
JAMA
J Am Board Fam Pract
J Am Coll Cardiol
J Am Coll Surg
J Am Geriatr Soc
J Clin Epidemiol
J Fam Pract
J Gen Intern Med
J Infect Dis
J Intern Med

J Neurol Neurosurg Psychiatry

$\mathrm{J}$ Pediatr

J Vasc Surg

Lancet

Med Care

Med J Aust

N Engl J Med

Neurology

Obstet Gynecol

Pain

Pediatrics

Rheumatology

Spine

Stroke

Surgery

Thorax

*Approximately 60 additional journals are reviewed. This list is available on request. 